\title{
Preface
}

\section{Critical Care and Oncology}

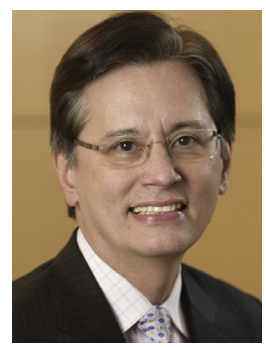

Stephen M. Pastores, MD, MACP, FCCP, FCCM

Editor

Cancer remains a leading health care challenge worldwide, including the United States, where 1.8 million new cases of cancer and 606,520 deaths from cancer are estimated in 2020. Over the past few decades, our understanding of the biology of cancer has grown rapidly, leading to major therapeutic advances in oncology, including the use of targeted agents, immune checkpoint blockade, and chimeric antigen receptor T-cell therapy, as well as innovations in cancer surgery and radiotherapy. These developments have improved not only patient outcomes but also their quality of life. However, oncology patients remain vulnerable and at high risk of developing acute critical illness requiring admission to the intensive care unit (ICU). Oncology patients account for up to $20 \%$ of all ICU patients. Many of these patients are admitted to the ICU due to either the malignancy itself, cancer treatment-related side effects or complications, or an underlying medical condition unrelated to the cancer.

Given the complex care needs of oncology patients who become critically ill, it has become increasingly clear that expert knowledge from specialists of several disciplines working collaboratively with ICU clinicians is mandatory to ensure the best possible outcomes and appropriate use of available critical care resources.

In this issue of Critical Care Clinics, an excellent group of highly regarded and practicing clinicians in the field of critical care oncology and supportive care has been assembled to provide their expertise on the care of the critically ill cancer patient. This issue leads off with important articles on the triage and prognostication of cancer patients admitted to the ICU and the key aspects of ICU organization and interdisciplinary care of these patients. The articles that follow highlight critical care issues in hematopoietic stem cell transplant recipients, diagnostic and management strategies of toxicities associated with immunotherapy and cardiotoxicity in the era of novel cancer therapies, infectious disease complications, and oncologic emergencies necessitating ICU care. The final article discusses the scope of the ethical conundrums that arise in the provision of end-of-life care to adult patients with cancer and the need for early 
involvement of palliative care teams in the critical care setting. It was a privilege and honor for me to work with them on this issue.

I would like to express my sincere appreciation and gratitude to all of the contributing authors and to Casey Marie Potter, Kerry Holland, Joanna Collett, and Nicholas Henderson at Elsevier for their assistance in getting this work product completed in the midst of the COVID-19 global pandemic.

Stephen M. Pastores, MD, MACP, FCCP, FCCM

Memorial Sloan Kettering Cancer Center

1275 York Avenue C-1179

New York, NY 10065, USA

E-mail address:

pastores@mskcc.org 